\title{
Corticosterone Circadian Secretion Differentially Facilitates Dopamine-mediated Psychomotor Effect of Cocaine and Morphine
}

\author{
Michela Marinelli, Pier Vincenzo Piazza, Véronique Deroche, Stefania Maccari, Michel Le Moal, and Hervé \\ Simon \\ Psychobiologie des Comportements Adaptatifs, INSERM U259, Université de Bordeaux II, 33077 Bordeaux Cedex, France
}

Studies of intravenous self-administration and psychomotor effects of drugs have recently suggested that stress-induced corticosterone secretion may be an important factor determining vulnerability to drugs of abuse. In this report, we studied if basal physiological corticosterone secretion modulates sensitivity to cocaine and morphine, and if changes in the reactivity of mesolimbic dopaminergic (DA) neurons, one of the principal substrates of drug-reinforcing effects, are involved. For this purpose we determined the psychomotor effects of these drugs in animals in which corticosterone secretion was suppressed by adrenalectomy and in adrenalectomized animals submitted to different cortlcosterone replacement therapies designed to mimic (1) only the diurnal levels of the hormone, obtained by the subcutaneous implantation of $50 \mathrm{mg}$ corticosterone pellets; (2) only the nocturnal levels, obtained by adding corticosterone (50 $\mu \mathrm{g} / \mathrm{ml}$ ) to the drinking solution during the dark period; and (3) the entire circadian fluctuation, obtained by combining the two previous treatments. Locomotor response to cocaine and morphine was studied after both systemic and central injections, into the nucleus accumbens for cocaine and into the ventral tegmental area for morphine. These sites were chosen because stimulant effects of cocaine and morphine injected in these structures are dopamine dependent. Our results show that suppression of corticosterone by adrenalectomy reduced the locomotor response to cocaine and morphine, injected both systemically and centrally. The reinstatement of diurnal levels of corticosterone totally reversed adrenalectomy's effects on the behavioral response to cocaine, whereas the reestablishment of the entire corticosterone circadian fluctuation (diurnal plus nocturnal levels) was necessary to reverse the response to morphine. In conclusion, our results indicate that physiological corticosterone secretion facilitates dopamine-mediated locomotor effects of cocaine and morphine. Since stimulant effects and activation of DA mesencephalic neurons by these drugs are related to their reinforcing properties, an interaction between corticosterone and dopamine may be part of the pathophysiological mechanism underlying vulnerability to drug abuse.

Received Aug. 31, 1993; accepted Oct. 19, 1993.

This work was supported by l'Institut National de la Santé et de la Recherche Médicale (INSERM) and l'Université de Bordeaux II. M.M. was supported by a grant of the Consiglio Nazionale delle Ricerche (CNR).

Correspondence should be addressed to Dr. Pier Vincenzo Piazza, INSERM U259, Rue Camille Saint-Saëns, 33077 Bordeaux Cedex, France.

Copyright (C 1994 Society for Neuroscience $0270-6474 / 94 / 142724-08 \$ 05.00 / 0$
[Key words: corticosterone, cocaine, morphine, dopamine, nucleus accumbens, ventral tegmental area, psychomotor effects, drug abuse]

Individual predisposition (De Wit et al., 1986; O'Brien et al., 1986) and life experiences (Piazza et al., 1991b) are two important factors influencing the development of drug addiction. Experimental studies have shown that although a higher vulnerability to drug intake is spontaneously observed in certain individuals, it can also be induced by life events and, in particular, by stress. For example, in the rat, only some subjects spontaneously develop amphetamine self-administration (Piazza et al., 1989, 1990b), whereas this behavior is enhanced by conditions such as social isolation (Alexander et al., 1978; Hadaway et al., 1979; Schenk et al., 1987; Bozarth et al., 1989), competition in the colony (Maccari et al., 1991), immobilization (Deroche et al., 1992b; Shaham et al., 1992), repeated tail pinch (Piazza et al., 1990a), and prenatal stress (Deminière et al., 1992). Individual vulnerability and stress-induced predisposition to drug intake are associated with an enhanced locomotor response to psychostimulants, opioids, and novelty (Piazza et al., 1989, 1990a,b; Hooks et al., 1991; Deroche et al., 1992a), as shown also by the increase in addictive properties of drugs observed when the locomotor effects of psychostimulants and opioids are sensitized (Lett, 1989; Piazza et al., 1990a; Gaiardi et al., 1991; Horger et al., 1991) by their repeated injections (for review, see Robinson and Becker, 1986; Kalivas and Stewart, 1991).

Several data have recently suggested that stress-induced corticosterone secretion may be an important factor determining both individual and stress-induced vulnerability to drug effects. First, predisposition to drug intake, spontaneously present in certain individuals or induced by stress in others, is associated with a longer-lasting corticosterone secretion in response to stress (Piazza et al., 1991a). Second, the administration of corticosterone, in the range of stress-induced levels, before self-administration increases the reinforcing properties of amphetamine (Piazza et al., 1991a). Third, suppression of stress-induced corticosterone secretion abolishes the sensitization to the psychomotor effects of morphine and amphetamine induced by stress (Deroche et al., 1992b, 1993), whereas repeated corticosterone administration, like repeated stress, sensitizes the locomotor response to amphetamine (Deroche et al., 1992a).

In this report, two main questions were addressed. First, may basal physiological corticosterone secretion, similarly to what has been observed for stress-induced secretion (Deroche et al., 1992a,b), modulate the behavioral sensitivity to the two main 
classes of drugs of abuse, psychostimulants and opioids? Second, is the influence of corticosterone on drug effects exerted through changes in the reactivity of mesolimbic dopaminergic (DA) neurons, one of the principal substrates of drug-reinforcing effects (for review, see Fibiger and Phillips, 1988; Koob and Bloom, 1988; Wise and Rompre, 1989; Le Moal and Simon, 1991)?

In order to answer these questions, we studied the locomotor response to the psychostimulant cocaine and to the opioid morphine. The two drugs were injected both systemically and centrally into the nucleus accumbens for cocaine and into the ventral tegmental area (VTA) for morphine. These sites were chosen because locomotor effects of cocaine (Kelly and Iversen, 1976; Delfs et al., 1990) and morphine (Joyce and Iversen, 1979; Kalivas et al., 1983; Vezina and Stewart, 1984) injected into these structures are dopamine dependent. Locomotor activity was investigated in animals completely lacking corticosterone or submitted to specific treatments designed to mimic the different phases of basal physiological corticosterone secretion. In rodents, plasma concentrations of this hormone are low and relatively constant during the diurnal period, which corresponds to the inactive period of these species, and rise to reach a peak during the first nocturnal hours (Akana et al., 1986). Three specific corticosterone substitutive therapies were used in order to reproduce (1) diurnal levels of the hormone, (2) nocturnal levels, or (3) the entire circadian fluctuation. The efficiency of these replacement therapies in mimicking the different phases of corticosterone physiological secretion was tested in the first experiment of this report.

\section{Materials and Methods}

Subjects. Male Sprague-Dawley rats (Iffa-Credo, Lyon, France) weighing 280-300 gm were used. Animals were individually housed with ad libitum access to food and water. A constant light/dark cycle (lights on from 6 A.M. to 8 P.M.) was maintained in the animal room, and temperature $\left(22^{\circ} \mathrm{C}\right)$ and humidity $(60 \%)$ were kept constant. Animals were allowed at least $I$ week of habituation to the animal room before starting experiments.

\section{General methods}

Locomotor activity and constitution of experimental groups. Locomotor activity was measured in a circular corridor $(10 \mathrm{~cm}$ wide and $70 \mathrm{~cm}$ in diameter). Four photoelectric cells placed at the perpendicular axis of this apparatus automatically recorded locomotion. Since it has been previously shown that locomotor response to novelty is correlated to sensitivity to the psychomotor effects of drugs (Piazza et al., 1989, 1991 b; Hooks et al., 1991), we ensured a homogeneous distribution of this factor throughout the different experimental groups. For this purpose, $1 \mathrm{~d}$ prior to being submitted to the surgery used for the different corticosterone replacement therapies, all animals were tested for their locomotor response to novelty. Hence, rats were placed in the circular corridor between 4 and 6 P.M. and evenly distributed among the different experimental groups according to their activity score cumulated over the $2 \mathrm{hr}$ of testing.

Adrenalectomy and corticosterone replacement therapies. Adrenalectomy was performed between 8 and 10 A.M. under ether anaesthesia via the dorsal approach. Following surgery, $\mathrm{NaCl}(0.9 \%)$ was added to the drinking water. Some of the adrenalectomized animals received one of three different corticosterone replacement therapies. The first replacement therapy was designed to mimic the basal diurnal levels of corticosterone. Thus, adrenalectomized animals in this group (diurnal levels group) were implanted with a subcutaneous solid pellet of corticosterone designed to provide a constant release of the hormone in the range of the diurnal basal levels. Such pellets contained $50 \mathrm{mg}$ of corticosterone, adjusted to $100 \mathrm{mg}$ with cholesterol (Meyer et al., 1979). The second corticosterone replacement therapy was used to reproduce the nocturnal levels of the hormone (nocturnal levels group). This was obtaincd by adding, from 7 P.M. to 8 A.M., corticostcrone $(50 \mu \mathrm{g} / \mathrm{ml})$ to the drinking solution of adrenalectomized animals. The third corti- costerone replacement therapy was intended to imitate the complete circadian rhythm of the hormone and thus combined the two above described treatments. Adrenalectomized animals in this group (circadian fluctuation group) were subcutaneously implanted with the corticosterone pellet and received the corticosterone-enriched solution during the dark period. Adrenalectomized animals that did not receive any replacement therapy constituted the ADX group. Control animals (sham group) underwent the same surgical procedure as the ADX animals except that the adrenals were not removed.

Stereotaxic implantation. Rats were anesthetized with sodium pontobarbital $(50 \mathrm{mg} / \mathrm{kg}$, i.p.) and placed in a stereotaxic apparatus (Kopf Instruments) with incisor bar $5.0 \mathrm{~mm}$ above the interaural line. Chronic bilateral guide cannulas ( 23 gauge stainless steel) positioned $2 \mathrm{~mm}$ above the final injection site were implanted according to the stereotaxic atlas of Pellegrino et al. (1979). The guide cannulas were secured in place with the use of stainless steel screws and dental cement. Removable 30 gauge stainless steel stylets were inserted in the guide cannulas to prevent clogging between treatments. The stereotaxic coordinates relative to bregma were $\mathrm{AP}+3.7, \mathrm{~L} \pm 1.6$, and $\mathrm{V}-5.8$ from the skull for the nucleus accumbens and $\mathrm{AP}-3.2, \mathrm{~L} \pm 0.5$, and $\mathrm{V}-5.7$ for the VTA. At the end of the experiments, cannula placements were verified histologically on $100 \mu$ thionin-stained coronal sections.

Drugs and drug administration. Corticosterone 21-hemisuccinate (Agrar, Italy) was used for all experiments. Cocaine $\cdot \mathrm{HCl}$ and morphine sulfate were used for both systemic and intracerebral injections. Concentrations of corticosterone and morphine are expressed as base. Drugs for systemic administration were dissolved in sterile $0.9 \% \mathrm{NaCl}$ solution and injected subcutaneously in a $1 \mathrm{ml} / \mathrm{kg}$ volume at the doses of $15 \mathrm{mg} /$ $\mathrm{kg}$ for cocaine and $2 \mathrm{mg} / \mathrm{kg}$ for morphine. Drugs for central administration were dissolved in a vehicle solution reproducing the electrolytic content of cerebrospinal fluid and containing $125 \mathrm{~mm} \mathrm{NaCl}, 1.2 \mathrm{mM}$ $\mathrm{CaCl}_{2}, 2.7 \mathrm{mM} \mathrm{KCl}, 1.0 \mathrm{~mm} \mathrm{MgCl}_{2}$, and buffered with $0.2 \mathrm{~mm}$ of $\mathrm{Na}_{2} \mathrm{HPO}_{4} / \mathrm{NaH}_{2} \mathrm{PO}_{4}$ at $\mathrm{pH}$ 7.4. Central injections for both drugs were performed bilaterally in unrestrained rats over a period of $90 \mathrm{sec}$ in a volume of $1 \mu \mathrm{l} / \mathrm{side}$. The injection cannulas ( 30 gauge stainless steel) descended $2 \mathrm{~mm}$ below the guide cannulas and were left in place for 60 sec both before and after the administration period. Cocaine was injected in the nucleus accumbens at a dose of $50 \mu \mathrm{g} / \mathrm{side}$. Its $\mathrm{pH}$ was adjusted to 7.2-7.4 with dilute $\mathrm{NaOH}$ before central administration. Morphine was injected in the VTA at a dose of $1 \mu \mathrm{g} / \mathrm{side}$.

Corticosterone assay. Blood for corticosterone assay was collected in heparinized tubes. Plasma, obtained after centrifugation, was stored at $-20^{\circ} \mathrm{C}$ until assay. Plasma corticosterone was measured by radioimmunoassay (RIA kit, ICN Biomedicals, Inc.) using a highly specific corticosterone antiserum with a detection threshold of $0.1 \mu \mathrm{g} / 100 \mathrm{ml}$.

\section{Procedures}

Experiment 1: effect of adrenalectomy and corticosterone substitutive therapies on plasma corticosterone levels. Animals $(n=84)$ were submitted to sham operation, adrenalectomy, or one of the three replacement therapies. The following five groups were thus constituted: sham $(n=15)$, ADX $(n=17)$, diurnal levels $(n=17)$, nocturnal levels $(n=$ 20 ), and circadian fluctuation $(n=15)$. Plasma corticosterone levels were determined 1 week after the start of these treatments. In order to determine both the diurnal and nocturnal corticosterone levels, half of the animals in each group were killed at 9 P.M., and the remaining half at 9 A.M. the following morning.

Experiment 2: effect of adrenalectomy and corticosterone substitutive therapies on locomotor response to systemic injection of cocaine or morphine. Animals $(n-101)$ were first submitted to sham operation, adrenalectomy, or one of the three replacement therapies. Five groups were thus constituted: sham $(n=24), \operatorname{ADX}(n=18)$, diurnal levels, $(n$ $=19$ ), nocturnal levels $(n=24)$, and circadian fluctuation $(n=16)$. One week after the start of these treatments, half of the animals in each group were tested for the locomotor response to cocaine, and the remaining half for the locomotor response to morphine. For this test, animals were placed in the circular corridor at 9 A.M. After a $2 \mathrm{hr}$ period of habituation, all animals were injected subcutaneously with saline. Two hours later, the animals were injected with either cocaine or morphine. Locomotor activity was recorded for $2 \mathrm{hr}$ after saline and cocaine and for $3 \mathrm{hr}$ after morphine. Scores were recorded over $10 \mathrm{~min}$ intervals for animals receiving cocaine and over $\mathbf{3 0 ~ m i n ~ i n t e r v a l s ~ f o r ~ t h o s e ~ r e c e i v i n g ~}$ morphine.

Experiment 3: effect of adrenalectomy and corticosterone substitutive therapies on locomotor response to intraaccumbens injection of cocaine. 


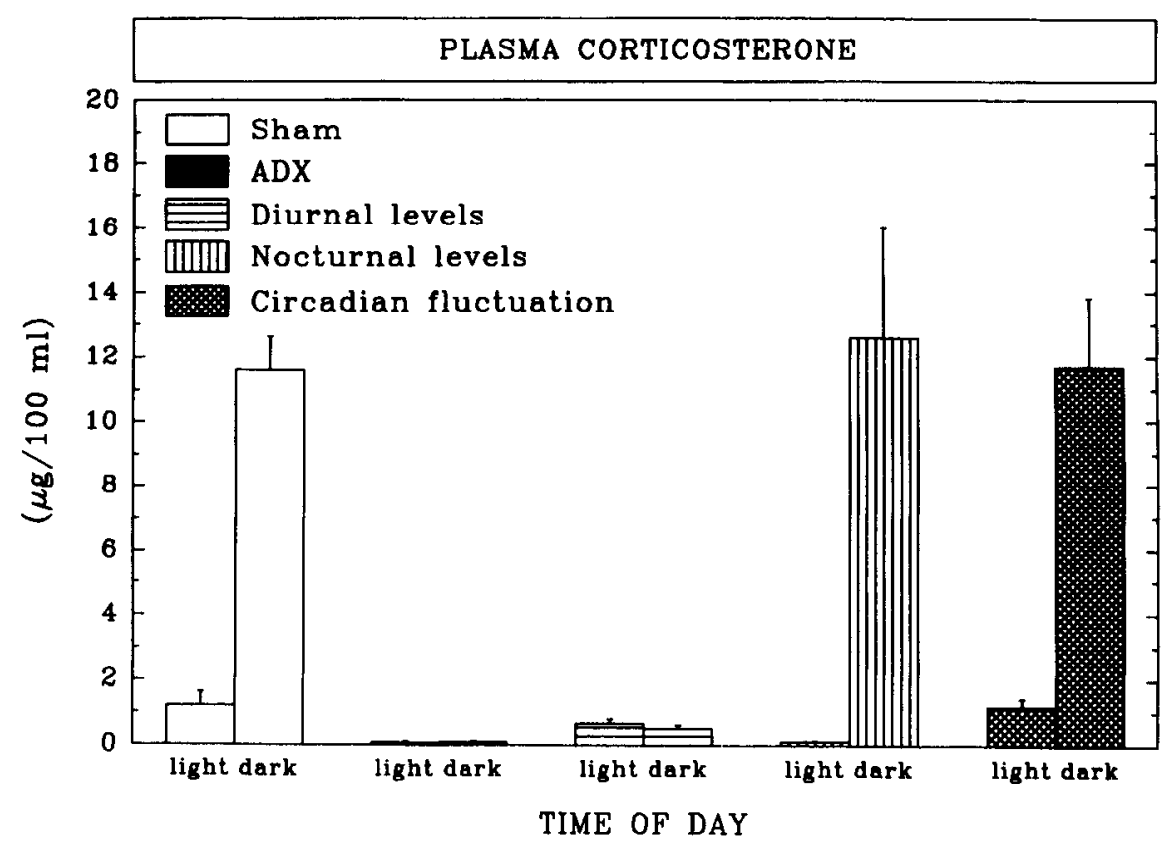

Figure 1. Effect of adrenalectomy and corticosterone replacement therapies on corticosterone plasma levels. In sham animals, corticosterone levels were higher in the dark period than in the light period. Corticosterone levels of the sham group in the light period were similar to those observed in the diurnal levels and circadian fluctuation groups killed at the same time. Corticosterone levels of the sham group in the dark period were similar to those of the circadian fluctuation and nocturnal levels groups killed at the same time. Adrenalectomized animals had lower corticosterone levels than all groups.

\author{
TIME OF DAY
}

Animals $(n=28)$ were first implanted with guide cannulas above the nucleus accumbens. Ten days later, they were submitted to either sham operation, adrenalectomy, or a corticosterone replacement therapy. Three experimental groups were thus constituted: sham $(n=10)$, ADX $(n=$ 9 ), and diurnal levels $(n=9)$. This substitutive therapy was chosen because the previous experiment revealed that corticosterone diurnal levels were sufficient to abolish the effect of adrenalectomy on the locomotor response to cocaine. One week later, the animals were tested on $2 \mathrm{~d}$ for the locomotor response to centrally injected vehicle and cocaine. The first day the animals were placed in the circular corridor at 10 A.M.; after a $2 \mathrm{hr}$ period of habituation, they were injected with the vehicle solution and their locomotor response recorded for $2 \mathrm{hr}$ over 10 min intervals. The second day the same procedure was repeated, but instead of receiving intraaccumbens vehicle, the animals were administered cocaine.

Experiment 4: effect of adrenalectomy and corticosterone substitutive therapies on locomotor response to intra-VTA injection of morphine. Animals $(n=20)$ were first implanted with guide cannulas aimed at the VTA. Ten days later they were submitted to either sham operation, adrenalectomy, or a corticosterone replacement therapy. Three experimental groups were thus constituted: sham $(n=7), \mathrm{ADX}(n=6)$, and circadian fluctuation $(n=7)$. This substitutive therapy was chosen because the experiment with systemic injection of morphine revealed that the complete circadian secretion was necessary to abolish the effects of adrenalectomy. One week later, the locomotor response to vehicle and morphine was determined by using the same procedure employed in the intraaccumbens cocaine experiment. Locomotor response to both substances was recorded for $3 \mathrm{hr}$ over $30 \mathrm{~min}$ intervals.

Statistical analysis. Analysis of variance (ANOVA) was used to analyze plasma levels of corticosterone and activity scores. NewmanKeuls test was used for post hoc analysis.

For experiment 1, the ANOVA was performed considering the different hormonal manipulations as first between factor (treatment, five levels), and the time of the day at which the blood sample was withdrawn as second between factor (time of the day, two levels). Single pairwise comparisons between treatments served to determine group differences.

For experiment 2, data were initially submitted to an ANOVA, which considered the different hormonal manipulations as between factor (treatment, five levels) and the time after the injection of either saline or drugs as within factor (time, 12 levels for saline and cocaine when considering the locomotor response to cocaine, and four levels for saline and six for morphine when considering the locomotor response to morphine). Subsequently, in order to better define the role of the different corticosterone replacement therapies on the response to each drug, a bifactorial analysis was performed over the four groups that were adrenalectomized (ADX, diurnal levels, nocturnal levels, and circadian fluctuation). The presence or the absence of subcutaneous corticosterone pellet was the first between factor. The presence or the absence of corticosterone-enriched solution was the second between factor. Thus, the first factor (pellet) determined the influence of the diurnal corticosterone levels while the second (corticosterone-enriched solution) ascertained the influence of the nocturnal corticosterone levels.

For experiments 3 and 4 , data were submitted to an ANOVA that considered the hormonal manipulations of each experiment as between factor (treatment, three levels), and the time after the injection of either the vehicle or the drug as within factor (time, 12 levels for vehicle and cocaine in experiment 3 , and six levels for vehicle and morphine in experiment 4).

\section{Results}

\section{Experiment 1: effect of adrenalectomy and corticosterone} substitutive therapies on corticosterone plasma levels

Adrenalectomy and the different corticosterone replacement therapies significantly modified plasma corticosterone levels [treatment effect, $F(4,74)=7.5, p<0.001$ ], and this effect was dependent on the time of day [treatment $\times$ time interaction, $F(4,74)=6.7, p<0.001$ ] (Fig. 1). In sham animals, corticosterone levels were higher during the dark period than the light phase $[F(1,13)=10.1, p<0.01]$. Adrenalectomy was able to constantly suppress endogenous corticosterone secretion. Corticosterone levels in ADX rats were lower than the minimal detection threshold of our assay $(0.1 \mu \mathrm{g} / 100 \mathrm{ml}$ of plasma) and did not differ between the light and dark period $[F(1,15)=0.1$, $p>0.8$. Implantation of corticosterone pellets constantly mimicked diurnal levels of the hormone. Thus, in adrenalectomized animals submitted to this replacement therapy (diurnal levels group), plasma corticosterone levels did not change over the day $[F(1,15)=0.7, p>0.4]$ and did not differ from levels observed in sham rats during the light cycle $[F(1,13)=1.5, p$ $>0.3$, but were lower than levels observed in sham rats during the dark period $[F(1,15)=15.2, p<0.01]$. Administration of corticosterone in the drinking solution specifically reproduced the nocturnal levels of the hormone. Thus, in the nocturnal levels group, the corticosterone levels during the dark phase did not differ from those observed in sham animals for the same period of the day $[F(1,16)=0.04, p>0.8]$, whereas the levels in the morning did not differ from those of $\mathrm{ADX}$ rats $[F(1,17)$ 


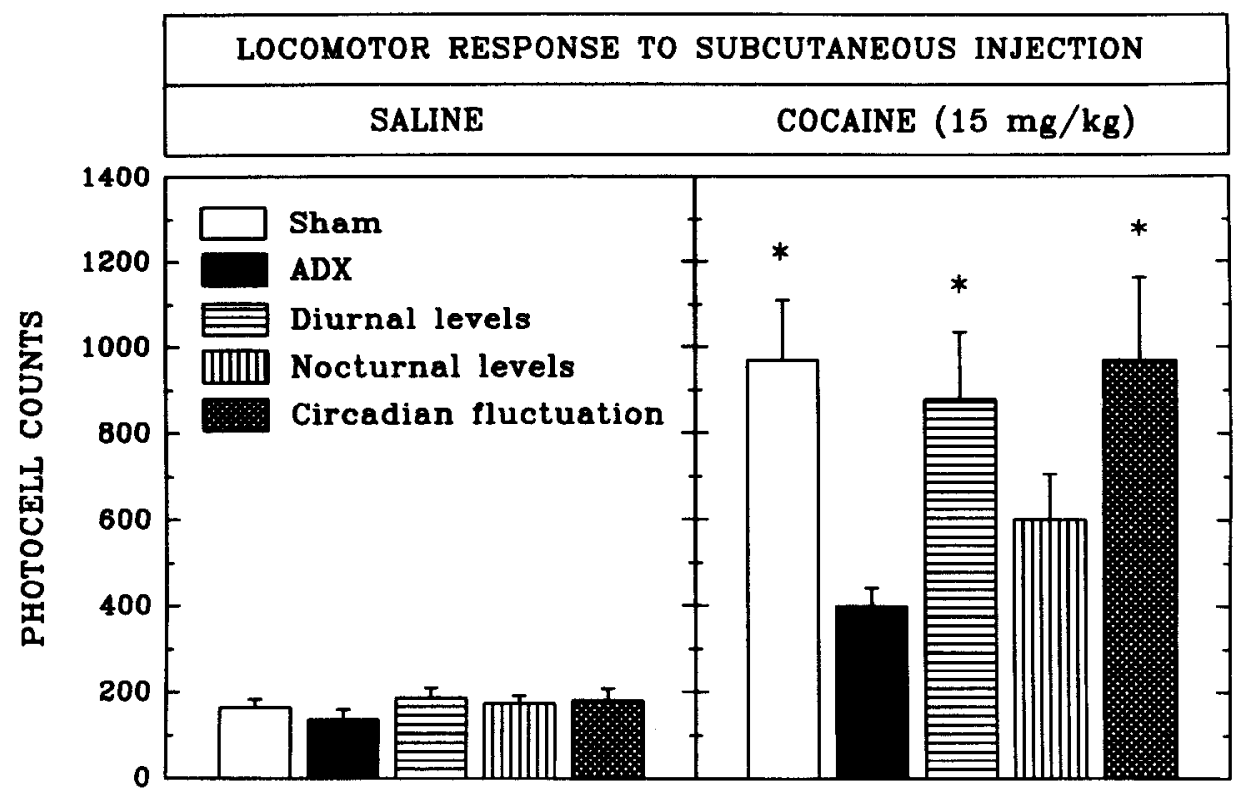

Figure 2. Effect of adrenalectomy and corticosterone replacement therapies on the locomotor response to systemic injection of saline and cocaine. No differences between the experimental groups were observed on the response to saline, whereas suppression of corticosterone secretion by adrenalectomy ( $A D X$ group) decreased the response to cocaine. This effect was abolished by reconstructing either the diurnal corticosterone levels (diurnal levels group) or the entire corticosterone circadian cycle (circadian fluctuation group), but not by restoring the nocturnal levels (nocturnal levels group). ADX animals did not differ from the nocturnal levels group, but exhibited lower locomotor scores compared to those observed in sham, diurnal levels, and circadian fluctuation groups $\left(^{*}, p<0.05\right)$. Furthermore, these last three groups did not differ in activity scores.
$=1.4, p>0.3]$. The association of the corticosterone pellet implantation with the administration of corticosterone in the drinking solution was able to mimic the corticosterone circadian secretion. Consequently, adrenalectomized animals submitted to this replacement therapy (circadian fluctuation group) did not differ from sham rats during either the dark period $[F(1,14)=$ $0.001, p>0.9]$ or the light phase $[F(1,12)=0.002, p>0.9]$.

\section{Experiment 2: effect of adrenalectomy and corticosterone substitutive therapies on locomotor response to systemic injection of cocaine or morphine}

Locomotor response to cocaine. Neither adrenalectomy nor the replacement therapies modified the locomotor response to saline [treatment effect, $F(4,46)=0.7, p>0.6$ ], although they affected the response to cocaine [treatment effect, $F(4,46)=3.2, p<$ 0.05 ] in a time-stable manner [treatment $\times$ time interaction, $F(44,506)=1.1, p>0.3$ ] (Fig. 2). Suppression of basal corticosterone levels reduced locomotor response to cocaine. Thus, ADX rats exhibited lower activity scores compared to those registered in sham animals $(p<0.05)$. Reestablishment of the corticosterone diurnal levels suppressed the effects of adrenalectomy. Animals in the diurnal levels group showed a higher motor response to cocaine than the one observed in ADX animals $(p<0.05)$, but not from the one of sham rats. The reproduction of the nocturnal levels of corticosterone alone was not sufficient to restore the decrease in the response to cocaine induced by adrenalectomy. In fact, there was no difference between the locomotor response of animals in the nocturnal levels group and that of ADX ones. Reestablishment of the complete circadian fluctuation did not improve the restoration of the locomotor response to cocaine already obtained by providing the animals with the diurnal levels alone. Thus, animals of the circadian fluctuation group showed a higher response than the one observed in ADX animals $(p<0.05)$ but did not differ from either the sham or the diurnal levels groups. The dependence of the psychomotor effects of cocaine on the diurnal levels of corticosterone was confirmed by the bifactorial analysis that considered the effect of the different replacement therapies in the four adrenalectomized groups. This analysis revealed a significant effect of corticosterone pellet implantation [pellet effect,
$F(1,35)=9.3, p<0.01]$, but not of the administration of corticosterone in drinking solution [corticosterone-enriched solution effect, $F(1,35)=1.6, p>0.2]$. Furthermore, no significant interaction was found between the two factors.

Locomotor response to morphine. Adrenalectomy and replacement therapies did not affect the response to saline [treatment cffcct, $F(4,45)=0.90, p>0.5$ ], but significantly altered the locomotor response to morphine [treatment effect, $F(4,45)$ $=6.6, p<0.001$ ] in a time-dependent manner [treatment $\times$ time interaction, $F(20,225)=2.0, p<0.01$ ] (Fig. 3). Suppression of corticosterone secretion reduced the stimulant effects of morphine. Thus, ADX animals showed lower activity scores than those displayed by sham rats $(p<0.01)$. Reproduction of either the diurnal levels or the nocturnal levels only partially reversed the effect of adrenalectomy. Thus, animals in both diurnal and nocturnal levels groups displayed a higher activity compared to the one of ADX rats ( $p<0.05$ in both cases), but still lower than the one observed in sham animals ( $p<0.05$ in both cases). Only the restoration of the entire corticosterone circadian secretion totally abolished the effects of adrenalectomy. Animals in the circadian fluctuation group showed a higher locomotor response to morphine compared to that of $\operatorname{ADX}$ rats $(p<0.01)$ and did not differ from the one observed in sham animals. The bifactorial analysis confirmed that both diurnal and nocturnal levels were necessary in order to abolish the effects of adrenalectomy. A significant effect was detected for both factors [pellet effect, $F(1,34)=10.8, p<0.01$; corticosterone-enriched solution effect, $F(1,34)=9.8, p<0.01]$.

\section{Experiment 3: effect of adrenalectomy and corticosterone substitutive therapies on locomotor response to intraaccumbens injection of cocaine}

The results obtained by intraaccumbens cocaine injections reproduced those observed after the systemic injection of the drug. Neither adrenalectomy nor the corticosterone replacement therapy used in this experiment significantly modified the locomotor response to intraaccumbens vehicle injection [treatment effect, $F(2,25)=2.0, p>0.1]$. In contrast, a significant effect of these treatments was observed on the locomotor response to cocaine [treatment effect, $F(2,25)=6.9, p<0.01$ ] (Fig. 4). Suppression 
Figure 3. Effect of adrenalectomy and corticosterone replacement therapies on the locomotor response to systemic injection of saline and morphine. The locomotor response to saline was not modified by the treatments; however, suppression of corticosterone secretion by adrenalectomy ( $A D X$ group) decreased the locomotor response to morphine. Only the reconstruction of the entire corticosterone cycle (circadian fluctuation group) was able to antagonize this effect completely. Accordingly, sham and circadian fluctuation animals expressed equivalent locomotor scores, and both these groups showed a higher response than ADX $(p<0.01$ in both cases), diurnal levels $(p<0.05$ in both cases), and nocturnal levels ( $p$ $<0.05$ in both cases) animals. In the diurnal levels and nocturnal levels groups only a partial, but significant ( $p$ $<0.05$ in both cases), recovery of the effects of adrenalectomy was observed.

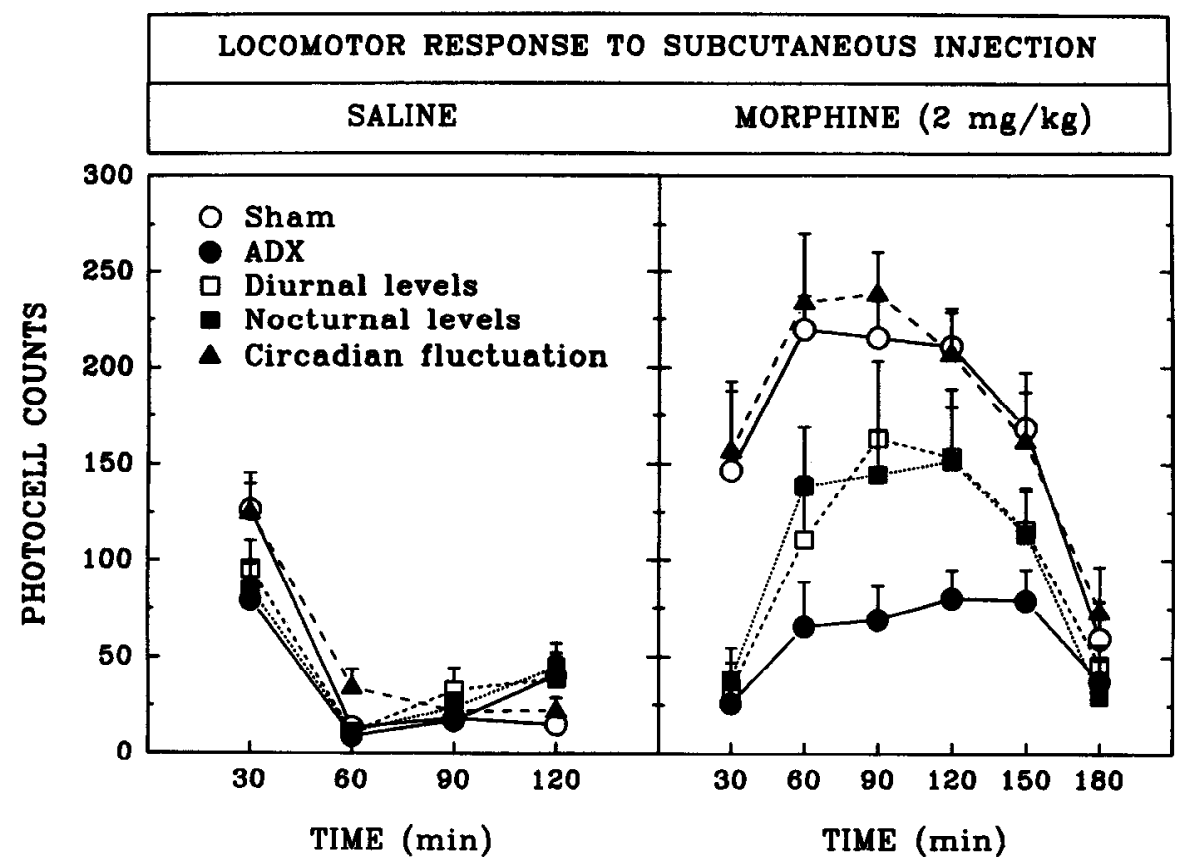

of corticosterone diminished the locomotor response to intraaccumbens cocaine. ADX animals showed activity scores lower than those of sham rats $(p<0.01)$. Furthermore, the restoration of the diurnal levels suppressed the effect of adrenalectomy. Animals in the diurnal levels group showed a higher locomotor response than that of $\mathrm{ADX}$ rats $(p<0.01)$ and did not differ from sham animals.

\section{Experiment 4: effect of adrenalectomy and corticosterone} substitutive therapies on locomotor response to intra-VTA injection of morphine

The results of this experiment reproduced the ones obtained after systemic administration of morphine. Neither adrenalectomy nor the replacement therapy used in this experiment significantly affected the locomotor response to intra-VTA vehicle injection [treatment effect, $F(2,17)=2.2, p>0.1$ ]. Significant effects on locomotor activity were, however, observed in response to intra-VTA morphine injection [treatment effect, $F(2,17)$ $=14.2, p<0.001$ ] (Fig. 5). Again, ADX animals showcd a lower locomotor response compared to the one observed in sham rats $(p<0.001)$, and the reinstatement of the entire corticosterone circadian secretion reversed this effect. Thus, in animals of the circadian fluctuation group, the locomotor response to morphine was higher than the one observed in ADX rats ( $p$ $<0.001$ ), but equivalent to the one of sham animals.

\section{Discussion}

Our results demonstrate that stimulant effects of cocaine and morphine depend on physiological basal levels of corticosterone and that this hormone facilitates the psychomotor properties of both drugs. Suppression of corticosterone by adrenalectomy reduced the locomotor response to the systemic or central injec-
Figure 4. Effect of adrenalectomy and restoration of diurnal corticosterone levels on the locomotor response to intraaccumbens vehicle and cocaine. Groups did not differ in the locomotor response to vehicle. Suppression of corticosterone levels by adrenalectomy ( $A D X$ group) reduced the locomotor response to cocaine and the reinstatement of basal diurnal levels of corticosterone reversed this effect. Thus, ADX animals exhibited lower activity scores than both sham $(* *, p<0.01)$ and diurnal levels $(* *, p<0.01)$ groups, and the latter two groups did not differ.

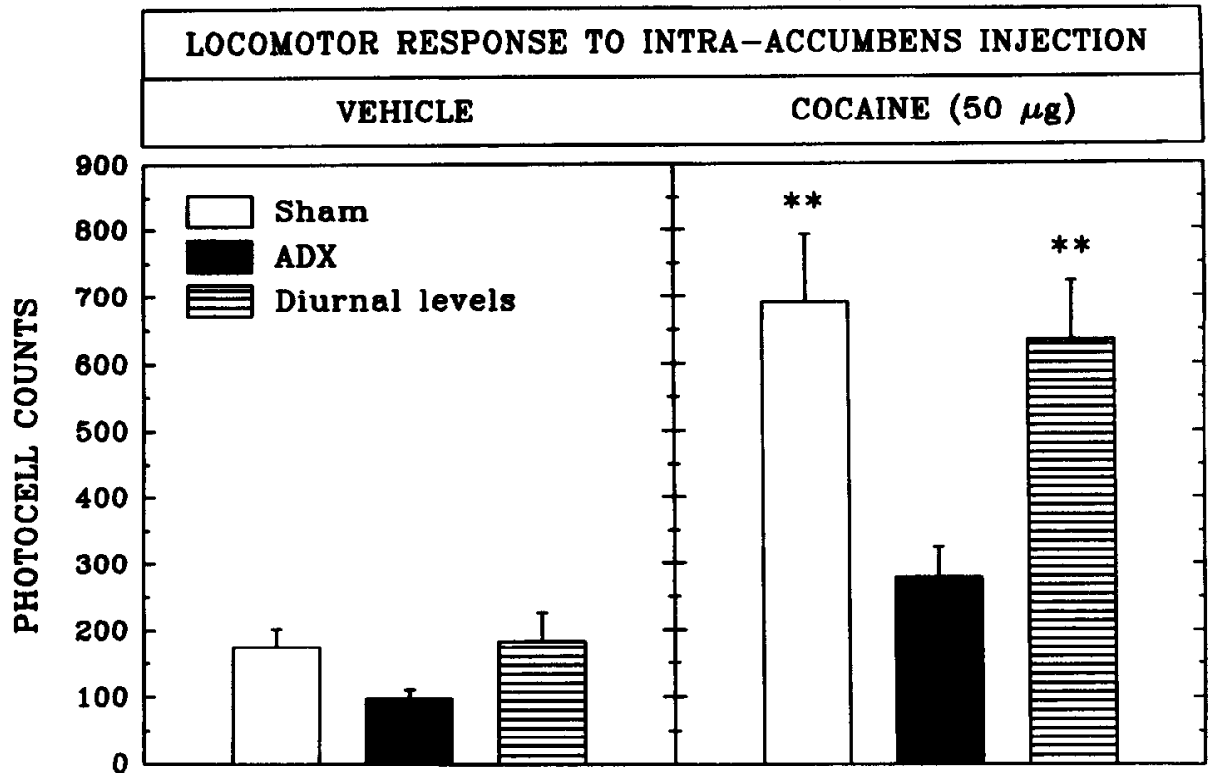




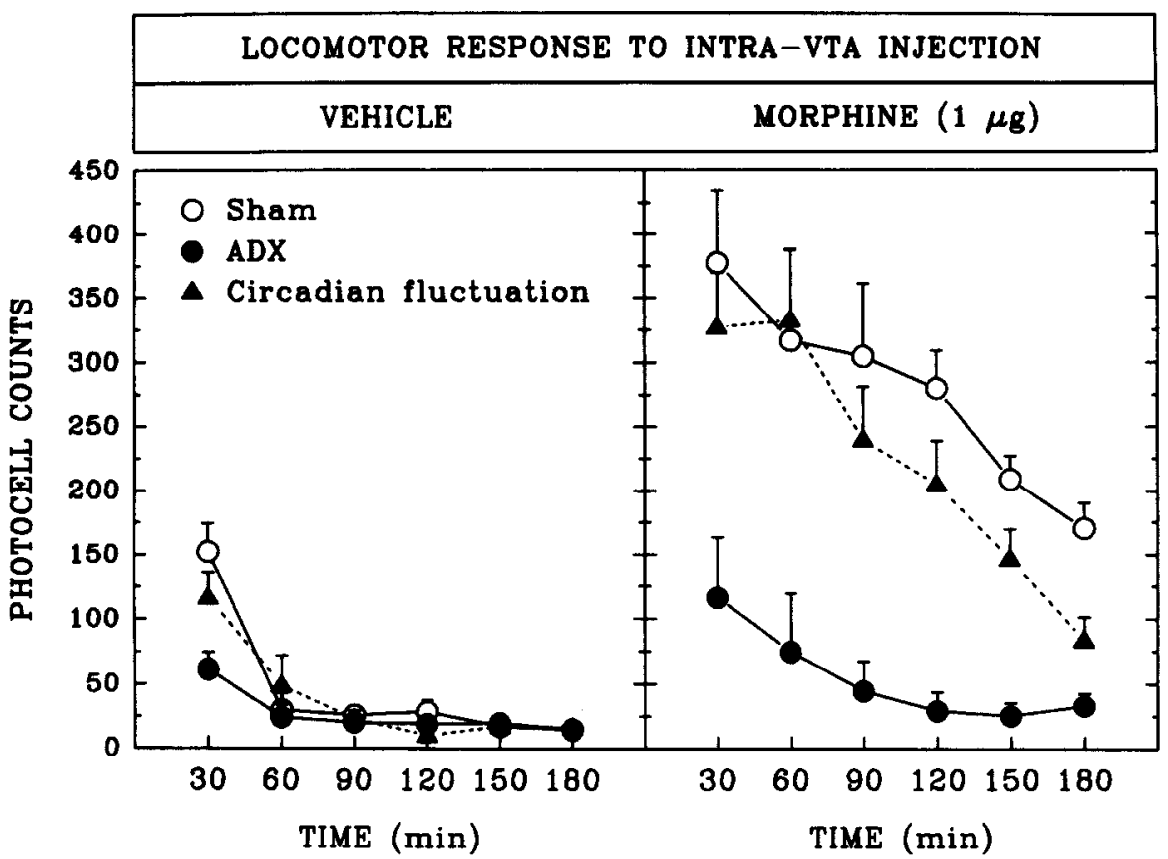

Figure 5. Effect of adrenalectomy and reconstruction of the entire corticosterone fluctuation on the locomotor response to intra-VTA vehicle and morphine. Groups did not differ in the locomotor response to vehicle. Suppression of corticosterone levels by adrenalectomy ( $A D X$ group) reduced the locomotor response to morphine and the reinstatement of the entire circadian secretion of corticosterone reversed this effect. Thus, ADX animals exhibited lower activity scores than both sham $(p<0.001)$ and circadian fluctuation groups $(p<0.001)$, whereas the latter two groups did not differ. tion of both cocaine and morphine. In contrast, corticosterone replacement therapies, mimicking physiological corticosterone secretion, totally reversed this effect.

Psychomotor effects of morphine and cocaine were differentially influenced by the different phases of corticosterone circadian secretion. Cocaine effects were dependent only on basal diurnal levels. For this drug, the effect of adrenalectomy was not significantly affected by restoration of the nocturnal peak, but was totally reversed by the substitutive therapy mimicking the diurnal levels. In contrast, the stimulant effect of morphine depended on the entire circadian corticosterone secretion, since the effect of adrenalectomy was only partially reversed by the reinstatement of diurnal or nocturnal levels but totally reversed by the substitutive therapy restoring both diurnal and nocturnal levels of the hormone. This finding may explain the circadian variation in the intensity of certain behavioral effects of opioids. Higher effects in the dark than in the light period have been demonstrated for morphine-induced feeding (Bhakthavatsalam and Leibowitz, 1986), lethal effects of morphine (Campos et al., 1983), and naloxone reversible pain sensitivity (Mc Givern and Bernston, 1980). These results highlight the important and different physiological roles of the phases of corticosterone circadian fluctuation. This aspect, often neglected, of glucocorticoid physiology may be crucial for the understanding of the functional effects of glucocorticoids on the CNS.

The effects of corticosterone on the locomotor response to cocaine and morphine appear to be mediated by changes in the reactivity of DA mesencephalic neurons to these drugs. Thus, the influence of adrenalectomy and corticosterone replacement therapies on the stimulant effects of cocaine and morphine was comparable when the drugs were injected systemically or centrally, in the nucleus accumbens for cocaine and in the VTA for morphine. Several studies have shown that motor activation induced by administration of cocaine in the accumbens (Delfs et al., 1990) or of morphine in the VTA (Joyce and Iversen, 1979; Vezina and Stewart, 1984) depends on the activation of DA A10 neurons projecting to the nucleus accumbens.

The dependency on corticosterone of dopamine-mediated psychomotor effects of cocaine and morphine supports previous works suggesting that endogenous corticosterone secretion may be involved in the predisposition to drug abuse. Mesencephalic DA neurons are considered an important substrate of drugreinforcing effects (Fibiger and Phillips, 1988; Koob and Bloom, 1988; Wise and Rompre, 1989; Le Moal and Simon, 1991) and a higher sensitivity to the stimulant effects of drugs has been associated with an increased vulnerability to their reinforcing properties (Piazza et al., 1989; Wise and Bozarth, 1989). Furthermore, the higher sensitivity to the reinforcing and stimulant effects of psychostimulants, spontaneously present in certain individuals or induced by stress in others, is positively correlated with a long-lasting corticosterone secretion in response to stress (Piazza et al., 1991a,b). Finally, corticosterone injection to subjects spontaneously resistant to develop amphetamine self-administration increases their propensity to acquire this behavior (Piazza et al., 1991a).

Although the effects of corticosterone on cocaine and morphine involve dopamine, the different sensitivity of the two drugs to the circadian variation of the hormone indicates the existence of different primary mechanisms of action. Two principal hypotheses may be drawn. First, different types of central corticosteroid receptors may be involved: type I receptors, which are almost totally saturated by diurnal levels of corticosterone (Reul and De Kloet, 1985; Reul et al., 1987), may mediate the effects of the hormone on cocaine; type II receptors, which are fully occupied only during the nocturnal peak (Reul and De Kloet, 1985; Reul et al., 1987), may be the substrate of the effects of corticosterone on morphine. Second, different neural substrates may be involved. The primary site of action of cocaine and morphine is, in fact, different. Cocaine is an indirect dopamine agonist that principally blocks dopamine reuptake (Reith et al., 1980; Ritz et al., 1987) and exercises its stimulant effect by acting on DA terminals, but not cell bodies. Conversely, morphine activation of DA neurons occurs in the VTA and results from the release of DA cells from an inhibitory GABA input (for review, see Kalivas and Stewart, 1991). This hypothesis is supported by the fact that corticosterone can act on both DA and opioid transmission. DA neurons have corticosteroid receptors (Härfstrand et al., 1986) and DA activity seems to be 
increased by glucocorticoid hormones (Rothschild et al., 1985; Mittleman et al., 1992). Proenkephalin mRNA in the striatum is decreased by adrenalectomy (Chao and McEwen, 1990), and the effects of enkephalin on hippocampal slices are potentiated by corticosterone (Vidal et al., 1986).

The action of corticosterone on the psychomotor effects of drugs may also involve other neurotransmitters, such as GABA, 5-HT, and excitatory amino acids. All these neurotransmitters can modulate dopamine-mediated responses to opioids and psychostimulants (Scheel-Krüger et al., 1981; Kalivas et al., 1989; Kelland et al., 1990; Pulvirenti et al., 1991), and are influenced by corticosterone. Glucocorticoids have been reported to modify the binding capacity of 5-HT receptors (Biegon et al., 1985; De Kloet et al., 1986; Martire et al., 1989) and GABA receptors (Majewska et al., 1986; Majewska, 1987, Sutanto et al., 1989) and to potentiate glutamatergic transmission (Tischler et al., 1988; Sapolsky, 1990).

In conclusion, our results indicate that physiological corticosterone secretion facilitates dopamine-mediated stimulant effects of both cocaine and morphine. Since, for these drugs, the stimulant effects and the activation of DA mesencephalic neurons are closely related to their reinforcing properties (Wise and Bozarth, 1989; Pulvirenti et al., 1991), our data support the hypothesis that an interaction between corticosterone and dopamine may be a pathophysiological mechanism underlying vulnerability to drug intake. Consequently, the understanding of the mechanisms by which these two biological systems interact may open new insights for the development of future therapeutic strategies of addiction.

\section{References}

Akana SF, Cascio CS, Du J-Z, Levin N, Dallman MF (1986) Reset of feedback in the adrenocortical system: an apparent shift in sensitivity of adrenocorticotropin to inhibition of corticosterone in the morning and evening. Endocrinology 119:2325-2332.

Alexander BK, Coambs RB, Hadaway PF (1978) The effect of housing and gender on morphine self-administration in rats. Psychopharmachology (Berlin) 58:175-179.

Bhakthavatsalam P, Leibowitz SF (1986) Morphine-elicited feeding: diurnal rhythm, circulating corticosterone and macronutrient selection. Pharmacol Biochem Behav 24:911-917.

Biegon A, Rainbow TC, McEwen BS (1985) Corticosterone modulation of neurotransmitter receptors in rat hippocampus: a quantitative autoradiographic study. Brain Res 332:309-314.

Bozarth MA, Murray A, Wise RA (1989) Influence of housing conditions on intravenous heroin and cocaine self-administration in rats. Pharmacol Biochem Behav 33:903-907.

Campos AE, Luijan M, Lopez. F, Figueroa-Hernandez JI, Rodriguez R (1983) Circadian variation in the lethal effects of morphine in the mouse. Proc West Pharmacol Soc 26:101-103.

Chao HM, McEwen BS (1990) Glucocorticoid regulation of proenkephalin messenger ribonucleic acid in the rat striatum. Endocrinology 126:3124-3130.

De Kloet ER, Sybesma H, Reul JMHM (1986) Selective control by corticosterone of serotonin 1 receptor capacity in raphe-hippocampal system. Neuroendocrinology 42:513-521.

De Wit $\mathrm{H}$, Uhlenhuth $\mathrm{EH}$, Johanson $\mathrm{CE}$ (1986) Individual differences in the reinforcing and subjective effects of amphetamine and diazepam. Drug Alcohol Depend 16:341-360.

Delfs JM, Schreiber L, Kelley AE (1990) Microinjection of cocaine into the nucleus accumbens elicits locomotor activation in the rat. $J$ Neurosci 10:303-310.

Deminière JM, Piazza PV, Guegan G, Abrous N, Maccari S, Le Moal M, Simon H (1992) Increased locomotor response to novelty and propensity to intravenous self-administration in adult offspring of stressed mothers. Brain Res 586:135-139.

Deroche V, Piazza PV, Maccari S, Le Moal M, Simon H (1992a) Repeated corticosterone administration sensitizes the locomotor response to amphetamine. Brain Res 584:309-313.
Deroche V, Piazza PV, Casolini P, Maccari S, Le Moal M, Simon H (1992b) Stress-induced sensitization to amphetamine and morphine psychomotor effects depend on stress-induced corticosterone secretion. Brain Res 598:343-348.

Deroche V, Piazza PV, Casolini P, Le Moal M, Simon H (1993) Sensitization to the psychomotor effects of amphetamine and morphine induced by food restriction depend on corticosterone secretion. Brain Res 611:352-356.

Fibiger HC, Phillips AG (1988) Mesocorticolimbic dopamine systems and reward. Ann NY Acad Sci 537:206-215.

Gaiardi M, Bartoletti M, Bacchi A, Gubellini C, Costa M, Babbini M (1991) Role of repeated exposure to morphine in determining its affective properties: place and taste conditioning studies in rats. Psychopharmacology (Berlin) 103:183-186.

Hadaway PF, Alexander BK, Coambs RB, Beyerstein B (1979) The effect of housing and gender on preference for morphine-sucrose solutions in rats. Psychopharmacology (Berlin) 66:87-91.

Härfstrand A, Fuxe K, Cintra A, Agnati LF, Zini I, Wilkström AC, Okret S, Yu ZY, Goldstein M, Steinbuch H, Verhofstad A, Gustafsson JA (1986) Glucocorticoid receptor immunoreactivity in monoaminergic neurons of rat brain. Proc Natl Acad Sci USA 238:302-322.

Hooks MS, Jones GH, Smith AD, Neill DB, Justice JB (1991) Response to novelty predicts the locomotor and nucleus accumbens dopamine response to cocaine. Synapse 9:121-128.

Horger BA, Shelton K, Schenk S (1991) Preexposure sensitizes rats to the rewarding effects of cocaine. Pharmacol Biochem Behav 37: 707-711.

Joyce EM, Iversen SD (1979) The effect of morphine applied locally to mesencephalic dopamine cell bodies on spontaneous motor activity in the rat. Neurosci Lett 14:207-212.

Kalivas PW, Stewart J (1991) Dopamine transmission in the initiation and expression of drug- and stress-induced sensitization of motor activity. Brain Res Rev 16:223-244.

Kalivas PW, Widerlöv E, Stanley D, Breese G, Prange AJ Jr (1983) Enkephalin action on the mesolimbic system: a dopamine-dependent and dopamine-independent increase in locomotor activity. J Pharmacol Exp Ther 227:229-237.

Kalivas PW, Duffy P, Barrow J (1989) Regulation of the mesocorticolimbic dopamine system by glutamic acid receptor subtypes. J Pharmacol Exp Ther 251:378-387.

Kelland MD, Freeman AS, Chiodo LA (1990) Serotoninergic afferent regulation of the basic physiology and pharmacological responsiveness of nigrostriatal dopamine neurons. J Pharmacol Exp Ther 253: 803-811.

Kelly PH, Iversen SD (1976) Selective 6-OHDA-induced destruction of mesolimbic dopamine neurons: abolition of psychostimulant-induced locomotor activity in rats. Eur J Pharmacol 40:45-56.

Koob GF, Bloom FE (1988) Cellular and molecular mechanisms of drug dependence. Science 242:715-723.

Le Moal M, Simon H (1991) Mesocorticolimbic dopamine network: functional and regulatory roles. Physiol Rev 71:155-234.

Lett BT (1989) Repeated exposures intensify rather than diminish the rewarding effects of amphetamine, morphine, and cocaine. Psychopharmacology (Berlin) 98:357-362.

Maccari S, Piazza PV, Deminière JM, Lemaire V, Mormède P, Simon $H$, Angelucci L, Le Moal M (1991) Life events-induced decrease of corticosteroid type I receptors is associated with reduced corticosterone feedback and enhanced vulnerability to amphetamine self-administration. Brain Res 547:7-12.

Majewska MD (1987) Antagonist-type interaction of glucocorticoids with the GABA receptor-coupled chloride channel. Brain Res 418: 377-382.

Majcwska MD, Harrison NL, Schwartz RD, Barker JL, Paul SM (1986) Steroid hormone metabolites are barbiturate-like modulators of the GABA receptors. Science 232:1004-1007.

Martire M, Pistritto G, Preziosi P (1989) Different regulation of serotonin receptors following adrenal hormone imbalance in the rat hippocampus. J Neural Transm 78:109-120.

Mc Givern RF, Bernston GG (1980) Mediation of diurnal fluctuations in pain sensitivity in the rat by food intake patterns: reversal by naloxone. Science 210:210-212.

Meyer JS, Micco DJ, Stephenson BS, Krey LC, McEwen BS (1979) Subcutaneous implantation method for chronic glucocorticoid replacement therapy. Physiol Behav 22:867-870.

Mittleman G, Blaha CD, Phillips AG (1992) Pituitary-adrenal and 
dopaminergic modulation of schedule-induced polydipsia: behavioral and neurochemical evidence. Behav Neurosci 106:402-408.

O'Brien CP, Ehrman RN, Terns JN (1986) Classical conditioning in human. In: Behavioral analysis of drug dependence (Goldberg SR, Stolerman IP, eds), pp 329-338. London: Academic.

Pellegrino LJ, Pellegrino AS, Cushman AJ (1979) A stereotaxic atlas of the rat brain. New York: Plenum.

Piazza PV, Deminière JM, Le Moal M, Simon H (1989) Factors that predict individual vulnerability to amphetamine self-administration. Science 245:1511-1513.

Piazza PV, Deminière JM, Le Moal M, Simon H (1990a) Stress- and pharmacologically-induced behavioral sensitization increases vulnerability to acquisition of amphetamine self-administration. Brain Res 514:22-26.

Piazza PV, Deminière JM, Maccari S, Mormède P, Le Moal M, Simon $\mathrm{H}$ (1990b) Individual reactivity to novelty predicts probability of amphetamine self-administration. Behav Pharmacol 1:339-345.

Piazza PV, Maccari S, Deminière JM, Le Moal M, Mormède P, Simon H (1991a) Corticosterone levels determine individual vulnerability to amphetamine self-administration. Proc Natl Acad Sci USA 88: 2088-2092.

Piazza PV, Deminière JM, Maccari S, Le Moal M, Mormède P, Simon H (1991b) Individual vulnerability to drug self-administration: action of corticosterone on dopaminergic systems as a possible pathophysiological mechanism. In: The mesolimbic dopamine system: from motivation to action (Willner P, Scheel-Krüger J, eds), pp 473-495. Chichester: Wiley.

Pulvirenti L, Swerdlow NR, Hubner CB, Koob GF (1991) The role of limbic-accumbens-pallidal circuitry in the activating and reinforcing properties of psychostimulant drugs. In: The mesolimbic dopamine system: from motivation to action (Willner P, Scheel-Krüger J, eds), pp 131-139. Chichester: Wiley.

Reith MEA, Shersen H, Lajtha A (1980) Saturable [ $\left.{ }^{3} \mathrm{H}\right]$-cocaine binding in central nervous system of mouse. Life Sci 27:1055-1062.

Reul JMHM, De Kloet FR (1985) Two receptor systems for corticosterone in rat brain: microdistribution and differential occupation. Endocrinology 117:2505-2511.

Reul JMHM, Van den Bosch FR, De Kloet ER (1987) Differential response of type I and type II corticosteroid receptors to changes in plasma steroid level and circadian rhythmicity. Neuroendocrinology 45:407-412.
Ritz MC, Lamb RJ, Goldberg SR, Kuhar MJ (1987) Cocaine receptors on dopamine transporters are related to self-administration of cocaine. Science 237:1219-1223.

Robinson TE, Becker JB (1986) Enduring changes in brain and behavior produced by chronic amphetamine administration: a review and evaluation of animal models of amphetamine psychosis. Brain Res Rev 11:157-198.

Rothschild AJ, Langlais PJ, Schatzberg AF, Miller MM, Saloman MS Lerbinger JE, Cole JO, Bird ED (1985) The effect of single acute dose of dexamethasone on monoamine and metabolites levels in the rat brain. Life Sci 36:2491-2505.

Sapolsky RM (1990) Glucocorticoids, hippocampal damage and the glutamatergic synapse. Prog Brain Res 86:12-23.

Scheel-Krüger J, Magelund G, Olianas MC (1981) Role of GABA in the striatal output system: globus pallidus, nucleus entopeduncolaris, substantia nigra and nucleus subthalamicus. In: GABA and the basal ganglia (Di Chiara G, Gessa GL, eds), pp 165-186. New York: Raven.

Schenk S, Lacelle G, Gorman K, Amit Z (1987) Cocaine self-administration in rats influenced by environmental conditions: implications for the etiology of drug abuse. Neurosci Lett 81:227-231.

Shaham Y, Alvares K, Nespor SM, Grundberg NF. (1992) Effect of stress on oral morphine and fentanyl self-administration in rats. Pharmacol Biochem Behav 41:615-619.

Sutanto W, Handelmann G, De Bree F, de Kloet ER (1989) Multifaceted interaction of corticosteroids with the intracellular receptors and with membrane GABA-A receptor complex in the rat brain. J Neuroendocrinol 1:243-247.

Tischler ME, Henriksen EJ, Cook PH (1988) Role of glucocorticoids in increased muscle glutamine production in starvation. Muscle Nerve 11:752-756.

Vezina P, Stewart J (1984) Conditioning and place-specific sensitization of increases in activity induced by morphine in the VTA. Pharmacol Biochem Behav 20:925-934.

Vidal C, Jordan W, Ziegelgänsberger W (1986) Corticosterone reduces the excitability of hippocampal pyramidal cells in vitro. Brain Res 383:54-59.

Wise RA, Bozarth MA (1989) A psychomotor stimulant theory of addiction. Psychol Rev 94:469-492.

Wise RA, Rompre PP (1989) Brain dopamine and reward. Annu Rev Psychol 40:191-225. 